\title{
Frequency-Domain Detection of Superluminal Group Velocity in a Distributed Bragg Reflector
}

\author{
Mohammad Mojahedi, Edl Schamiloglu, Kamil Agi, and Kevin J. Malloy
}

\begin{abstract}
Using a free-space configuration and a frequency-domain detection setup, group velocities of electromagnetic waves in a distributed Bragg reflector are investigated. Experimental data indicates that, near the regions of minimal transmission in our configuration, the group velocity is 2.1 times faster than the speed of light in vacuum. A transmission model based on diagonalization of the transfer matrix is used to compare the experimental data and the theoretical calculations, and good agreement between theory and experiment is obtained. An overview of the experimental uncertainties and their effects on the measured quantities is provided.
\end{abstract}

Index Terms-Electromagnetic fields, electromagnetic propagation in a dispersive medium, mirrors, superluminal propagation, velocity measurement.

\section{INTRODUCTION}

$\mathbf{T}$ HE QUESTION of electron tunneling time through a potential barrier has received much attention $[1$, and references therein]. In 1932, MacColl [2] argued that a transmitted wave packet tunneling through a potential barrier would appear on the other side of the barrier almost instantaneously. Using the method of stationary phase, Wigner [3] later showed that MacColl's conclusion was incorrect and that there was a finite time delay for the tunneling process. Wigner's analysis indicated that in the classical theory this delay or "retardation" expressed in units of distance must always remain larger than twice the radius of the scattering potential. However, Wigner also noted that, due to the wave nature of the wave function, the aforementioned inequality "will be violated occasionally ${ }^{1}$." Later, in a paper that re-ignited the most recent debate on superluminality and tunneling, Hartman [4] put MacColl's argument on more solid ground. His analysis implied that, for a sufficiently thick barrier (opaque barrier limit), the tunneling time was indeed shorter than the time required to traverse the same distance in vacuum, hence the tunneling velocity was superluminal (exceeding the speed of light in vacuum $c$ ).

More recently, possibly because of technical difficulties in conducting an experiment measuring the tunneling time for electrons, attention has turned to the analogous problem of electromagnetic propagation via evanescent modes through photonic barriers (photon tunneling) [5]. Since the "analogies"

Manuscript received September 22, 1999; revised December 13, 1999. This work was supported in part by the Army Research Office under Grant DAAH04-96-1-0439.

The authors are with the Center for High Technology Materials and the Department of Electrical and Computer Engineering, University of New Mexico, Albuquerque, NM 87106 USA.

Publisher Item Identifier S 0018-9197(00)02697-X.

${ }^{1}$ It is precisely these occasional violations that are our subject of interest. between photon tunneling and electron tunneling in particular [6], [7], and between the Maxwell-Helmholtz and Schrödinger wave equations in general [8] are well established, in principle, an experiment involving electromagnetic propagation can shed light on the more difficult problem of electron tunneling time.

Regardless of the aforementioned analogies, the subject of photonic tunneling time deserves its own consideration. With the operation of so many devices depending on photonic barriers such as distributed Bragg reflectors (DBR's), an inquiry regarding the ultimate limits on the speed of these devices as related to the phenomenon of photon tunneling is important. For example, at the present time, the use of DBR's in the design and implementation of vertical-cavity surface-emitting lasers (VCSEL's) [9], [10], surface-normal Fabry-Perot modulators [11], dynamic single-mode lasers [12], corrugated dielectric waveguides [13], and doubled-chirped mirrors [14] is a common practice. In this respect, the time associated with an electromagnetic wavepacket tunneling through a DBR is not only of scientific interest, but also of technological significance.

In the optical regime, using conjugate pairs of photons emitted simultaneously in the process of spontaneous parametric down-conversion, Chiao and coworkers [15] have found the tunneling velocity for a single photon through a DBR [also referred to as a one-dimensional photonic crystal (1DPC)] to be superluminal. In this quantum-domain measurement, they observed superluminal velocities 1.7 times faster than $c$. Similarly, Spielman et al. have used a Ti:sapphire laser capable of generating 10-15-fs optical pulses at $\lambda=0.8 \mu \mathrm{m}$ to study tunneling through a DBR [16]. This delicate experiment, which required "extremely high stability of the pulse parameter," took advantage of mirror-dispersion control to attain the required stability. Using a nonlinear, background-free correlation technique for the delay measurements, they were able to measure advances up to $6 \mathrm{fs}$ in the autocorrelated signal.

To obtain larger advances in time (nanoseconds), tunneling experiments can be performed in the microwave regime. In a series of experiments including undersized waveguides, two side-by-side prisms (frustrated total internal reflection), and slightly misaligned horn antennas, Ranfagni and his coworkers have investigated the phenomena of superluminal tunneling through photonic barriers [17]-[19].

Another series of microwave experiments were performed by Nimtz et al. [20]-[25]. Expanding on the work of Ranfagni et $a l$., they studied propagation through an undersized waveguide in both the frequency and time domain. Using the "Line-Reflect-Match" waveguide calibration technique, they were able to improve on previous results, allowing them to better measure superluminal group velocities. 
Nimtz and Enders also briefly considered transmission through a microwave DBR inserted inside an undersized waveguide. Although by their own admission the superluminal barrier crossing took place through both the DBR and the dispersive undersized waveguide [24], in this work and later recitation of this experiment [25], they have assigned the superluminal propagation solely to the DBR. The fact that tunneling took place through both structures means that the evanescent modes necessary for superluminal propagation are the hybrid modes of the undersized waveguide and the DBR. ${ }^{2}$ Moreover, in [24], [25], and, in fact, in all of their frequency-domain analysis, they used the Fourier transform to extend the frequency-domain network analyzer (NA) results to the time domain. This required an artificial assumption for the incident microwave wave packet, since the NA and its synthesized CW sweep-generator are incapable of generating incident microwave wave packets. Furthermore, in their use of the Fourier integral, they had to replace the $-\infty$ to $+\infty$ limits of the integral with the bounded limit of $v_{1}$ to $v_{2}$. This meant that their assumed incident pulse had zero frequency components outside the $\left(v_{1}, v_{2}\right)$ frequency interval $(8.7 \pm 0.5 \mathrm{GHz}$ in their particular case [24]). This simple point can cause many misinterpretations when presenting frequency-domain results as direct time-domain measurements, particularly in light of the fact that these neglected high-frequency components are essential in understanding the luminal Sommerfeld forerunners [26]. Finally, in their analysis, they only used the response function (transmission function) amplitude to calculate the time-domain signal. This assumed a constant phase for the response function, which is strictly true only for an infinitely long, undersized waveguide or DBR and, in fact, is erroneous in the case of the DBR with a limited number of periods, as will be seen in Section III. In light of the above, a correct and reliable measurement of the tunneling times and group velocities for DBR's is in order.

To understand the propagation through a finite, dispersive structure such as a DBR, the concept of group delay, defined as the frequency derivative of the transmission phase, is a better approach than the problematic Fourier transform construct alluded to earlier. However, to use the group delay approach, $a c$ curate and reliable measurements of the transmission phase are required, a task that surprisingly up to now has not been reported for a dispersive structure of this type. While acquiring the correct phase information for short optical pulses such as those used in the Spielman's experiment [16] is no easy task [27], [28], the correct use of a vector network analyzer (VNA) can make this task easier at microwave frequencies. Furthermore, because DBR dimensions scale with wavelength, performing the experiment at microwave frequencies leads to larger values for the group delay (hundreds of picoseconds) as compared to the optical frequencies (a few femtoseconds). This means that, for mi-

\footnotetext{
${ }^{2}$ As an illuminating example, let us cite an analogous situation described by Hauge [1]; he writes ". . . consider a packet impinging on a double barrier with two resonances of different life times. For a packet broad enough in its energy distribution to cover both resonances, a characterization of the tunneling delay in terms of a single (average) transmission time is, if formally possible, not very meaningful from a physical point of view."
}

crowave frequencies, direct observation (as opposed to autocorrelation) of the superluminal wave packet is possible [26].

Although while using a VNA one can measure the transmission coefficient magnitude and phase directly, care must be observed that the measured response is only due to the DBR. In other words, the DBR must be inserted into a free-space setup (not an undersized waveguide) and the response of the DBR must be properly de-embedded from the surrounding environment, a procedure commonly referred to as free-space or noncoaxial calibration. Furthermore, since the divergence of the electromagnetic waves are more pronounced at microwave frequencies, one must make an effort to collimate the quasi-CW sweeps of the VNA prior to its interaction with the DBR.

This paper is organized as follows. In Section II, a mathematical model based on diagonalization of the transfer matrix is developed. This approach expresses the transmission and reflection coefficients, along with the group delay and group velocity, in terms of the one-period matrix eigenvalues, which, in turn, allows expedient numerical evaluation of these quantities. Section III describes the free-space calibration technique and the experimental procedures and presents the measurement results. Section IV contains an analysis of the errors and experimental uncertainties, which reinforce the validity of the measured quantities. In Section V, some general remarks regarding superluminality and causality are given.

\section{TRANSFER MATRIX TECHNIQUE}

Fig. 1 depicts a DBR consisting of alternating layers of the dielectric Eccostock and air. The structure exhibits frequency regions for which transmission is prohibited (bandgaps) and frequency intervals for which transmission is allowed (passbands). It is for signals within the bandgaps of photonic crystals (PC's) that superluminal group velocities are expected.

In order to calculate the transmission properties of the finitelength DBR, standard transfer matrix techniques are followed [29]. The one-period matrix $\mathbf{M}$ is given by

$$
\mathbf{M}=\frac{1}{1-\rho_{i, j}^{2}}\left[\begin{array}{cc}
a & b \\
b^{*} & a^{*}
\end{array}\right]
$$

where

$$
\begin{aligned}
& a=\exp \left[-i\left(\beta_{i}+\beta_{j}\right)\right]-\rho_{i, j}^{2} \exp \left[i\left(\beta_{j}-\beta_{i}\right)\right] \\
& b=-\rho_{i, j} \exp \left[i\left(\beta_{i}-\beta_{j}\right)\right]+\rho_{i, j} \exp \left[i\left(\beta_{j}+\beta_{i}\right)\right]
\end{aligned}
$$

with

$$
\beta_{k}=\frac{2 \pi}{\lambda_{0}} n_{k} d_{k} \cos \left(\theta_{k}\right), \quad k=i \text { or } j .
$$

Here, $\lambda_{0}$ is the free-space wavelength, $\theta_{i}$ is the incident angle measured from the normal to the interface, $\rho_{i, j}$ is the TE or TM Fresnel reflection coefficient, $n_{k}$ is the index of refraction, and $d_{k}$ is the thickness of the $i$ or $j$ region. Although conservation of energy requires $\mathbf{M}$ to be unimodular $[\operatorname{det}(\mathbf{M})=1], \mathbf{M}$ is not unitary [30].

To calculate the transmission coefficient for a DBR consisting of $N$ dielectric slabs, $\mathbf{M}$ must be raised to the $N$ th power. While 


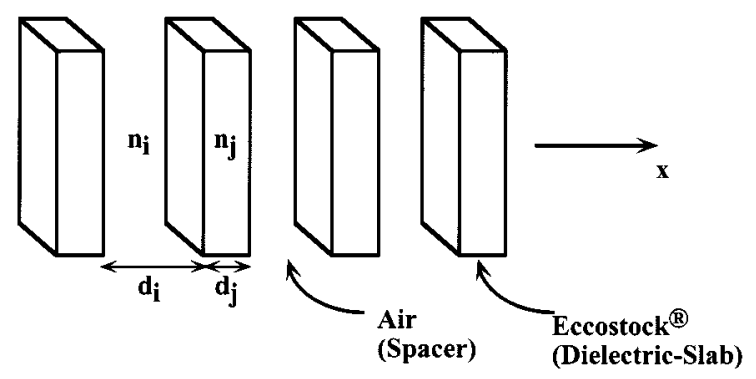

Fig. 1. Schematic of a DBR, also known as a 1DPC.

it is possible to write the resultant matrix in terms of Chebyshev polynomials [30], [31], here, using the diagonalization technique, the final result is expressed in terms of the eigenvalues and eigenvectors of the one-period matrix as [32]

$$
\mathbf{M}^{N}=\mathbf{S} \Lambda^{N} \mathbf{S}^{-1}
$$

where $\Lambda$ is the diagonal matrix of eigenvalues and $\mathbf{S}$ is the matrix of eigenvectors of $\mathbf{M}$. In writing (5), it is only required that $\mathbf{M}$ have a complete set of eigenvectors (although the eigenvalues can be degenerate). Equation (5) reduces the problem of raising a matrix to the $N$ th power to raising a scalar to the same power, hence decreasing the computational efforts. More importantly, since the eigenvalues and eigenvectors of $\mathbf{M}$ are known, a closed-form expression for $\mathbf{M}^{N}$, and, consequently, the transmission and reflection coefficients, can be obtained as given below:

$$
\begin{aligned}
& t=\frac{\left(\lambda_{1}-\lambda_{2}\right) e^{i \beta_{i}}\left(1-\rho_{i, j}^{2}\right)^{N}}{\lambda_{2}^{N}\left(a-\lambda_{2}\right)-\lambda_{1}^{N}\left(a-\lambda_{1}\right)}=|t| e^{i \varphi} \\
& r=\frac{\left(\lambda_{1}^{N}-\lambda_{2}^{N}\right) b}{\lambda_{1}^{N}\left(\lambda_{1}-a\right)-\lambda_{2}^{N}\left(\lambda_{2}-a\right)}=|r| e^{i \phi}
\end{aligned}
$$

where $\lambda_{1}$ and $\lambda_{2}$ are the eigenvalues of $\mathbf{M}$, expressed as

$$
\begin{aligned}
\lambda_{1,2}= & \left(1-\rho_{i, j}^{2}\right)^{-1} \times\left[\cos \left(\beta_{j}+\beta_{i}\right)-\rho_{i, j}^{2} \cos \left(\beta_{j}-\beta_{i}\right)\right. \\
& \pm \sqrt{\left.\left[\cos \left(\beta_{j}+\beta_{i}\right)-\rho_{i, j}^{2} \cos \left(\beta_{j}-\beta_{i}\right)\right]^{2}-\left(1-\rho_{i, j}^{2}\right)^{2}\right]}
\end{aligned}
$$

with the plus sign for $\lambda_{1}$ and the minus sign for $\lambda_{2}$. Equations (6) and (7) are unchanged if $n_{i}$ or $n_{j}$ are complex; hence, material losses can easily be taken into account.

Having acquired complex transmission and reflection coefficients, the transmission and reflection group delays now can be calculated as

$$
\begin{gathered}
\tau_{g}=-\frac{\partial \arg (t)}{\partial \omega}=-\frac{\partial \varphi}{\partial \omega} \\
\tau_{g}^{r}=-\frac{\partial \arg (r)}{\partial \omega}=-\frac{\partial \phi}{\partial \omega} .
\end{gathered}
$$

It can be shown that the envelope of a modulated electromagnetic pulse propagating through a linear and causal medium such as a DBR is delayed by an amount of time equal to the group delay as given by (9) [33], [26]. In this sense, one can define the velocity of propagation of the envelope through the medium as the "generalized group velocity," given in terms of the group delay as [33]

$$
v_{g}=\frac{L_{p c}}{\tau_{g}}
$$

where $L_{p c}$ is the total physical length of the finite DBR and is given by

$$
L_{p c}=(N-1)\left(d_{i}+d_{j}\right)+d_{j}
$$

This concept of generalized group velocity can also be arrived at by an ad hoc extension of the method of stationary phase. For real wavevectors, the derivative of the transmission phase, given by $\varphi=-k L_{p c}$, can be written as

$$
\tau_{g}=-\frac{\partial \varphi}{\partial \omega}=\frac{L_{p c}}{\partial \omega / \partial k}=\frac{L_{p c}}{v_{g}}
$$

which is the same as (11). Moreover, in a recent time-domain experiment with microwave wave packets (as opposed to $\mathrm{CW}$ sweeps of VNA presented here), it is observed that group delay and (11) accurately describe the propagation of the wave packet envelope through a DBR designed to have its mid-gap frequencies to coincide with the main frequency component of the incident wave packet [26]. These results will be published elsewhere.

In order to directly compare the delay time associated with a DBR of length $L_{p c}$ and the amount of the time required to travel the same distance in vacuum, one may divide both sides of (11) by $c$, giving ${ }^{3}$

$$
\frac{v_{g}}{c}=\frac{L_{p c}}{c \tau_{g}}=\frac{t_{0}}{\tau_{g}}
$$

where the definition of $t_{0}$ is self-evident from the above expression.

An interesting phenomenon occurs for frequencies tuned to the mid-gap of the DBR (region of minimal transmission). As will be seen in the next section, for these frequencies, the group delay $\left(\tau_{g}\right)$ is less than $t_{0}$, the time to transverse $L_{p c}$ in vacuum, hence this result can be described in terms of a group velocity exceeding the speed of light in vacuum. Two important points must be emphasized here. First, despite these superluminal group velocities, no genuinely new information has propagated faster than $c$. In other words, since under all circumstances the earliest part of any signal (known as the Sommerfeld forerunner) must remain exactly luminal, there is no violation of Einstein causality. Second, since most practical detection systems are designed to respond to the peak or half-maximum (the envelope) of a propagating pulse, and not to the high frequency and small amplitude of the Sommerfeld forerunner [26], [34], the aforementioned superluminal behavior may provide some practical benefit when signal latency is of concern.

\section{EXPERIMENTAL PROCEDURE}

Fig. 2 shows the free-space experimental setup. It consists of two K-band standard horn antennas (SHA's), connected to

${ }^{3}$ Equally well, (14) can be understood as a definition for normalized reciprocal group delay $\left(t_{0} / \tau_{g}\right)$. 
ports 1 and 2 of an HP-8722D VNA, configured to measure the transmission coefficient $\left(t\right.$ or $\left.S_{21}\right)$. The setup is enclosed in an anechoic chamber to reduce stray signals.

Extreme care must be taken to reduce systematic errors. For example, in order to minimize edge diffraction, Eccostock slabs with large transverse dimensions $(31 \mathrm{~cm} \times 31 \mathrm{~cm})$ along with a pair of microwave collimating and focusing lenses are used. In addition to diffraction, there are at least two other sources of errors: the effects of the experimental components such as the SHA's and lenses on the measurement, and the loss in the detected signal due to the propagation and the transmitter/receiver misalignment.

With recent advances in noncoaxial (free-space) calibration techniques for the VNA [35], [36], such as the "Thru-Line-Reflect" (TRL), it is possible to systematically remove the influence of the microwave components on the measured transmission coefficient. In this case, the "Thru" standard is a free-space transmission line of length $58.9 \mathrm{~cm}$, while the "Line" standard is a free-space transmission line of length $59.24 \mathrm{~cm}$. The "Reflect" is a copper plate set midway between transmitter and receiver. Here, the "Thru" is used to set the reference plane from which the transmission loss $(|t|)$ and insertion phase $(\varphi)$ will be measured. Performing the TRL calibration in free-space allows measuring the transmission coefficient solely due to the DBR and eliminates dispersion and dissipation losses associated with inserting the DBR inside a waveguide.

After calibrating the system (without the DBR), a reference plane of unit magnitude for $|t|$ and zero phase for $\varphi$ is established midway between the two SHA's. At this point, the DBR is inserted and the receiver horn is moved back exactly by $L_{p c}$. Clearly, moving the receiver horn backward introduces some propagation losses. This small loss $\left(t^{\text {prop }}\right)$ can be measured and its contribution can be deconvolved from the measured response according to

$$
t^{\text {measured }}(\omega)=t^{p c}(\omega) t^{\mathrm{prop}}(\omega)
$$

where $t^{p c}$ is the desired response function. To precisely control the backward movement of the receiver antenna, the insertion phase introduced by traveling the distance $L_{p c}$ can be monitored. By averaging traces, phase variations as small as one degree can easily be detected. From (16) below, it is clear that, at $21.5 \mathrm{GHz}$, a phase error of one degree corresponds to accuracy of $0.004 \mathrm{~cm}$ in length.

$$
\Delta z=\frac{\Delta \varphi c}{2 \pi v} .
$$

Fig. 3 shows the calculated transmission coefficient magnitude and phase $\left[\mid t^{p c}(\omega), \varphi\right]$, and the raw (nonsmoothed) measured values for a DBR with $N=3$.

Despite the fact that the signal amplitude has decreased by a factor of 20 in the bandgap, experiment and theory agree quite well. The average index of refraction for Eccostock was measured to be 3.4 - i 0.002 over the frequency range of interest. The thickness of the Eccostock slab and the air-spacer was determined to be 1.33 and $1.76 \mathrm{~cm}$, respectively. The above values were used throughout this paper in calculating the theoretical

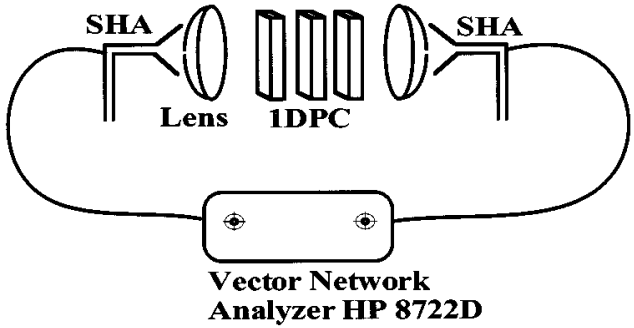

Fig. 2. Free-space experimental setup.

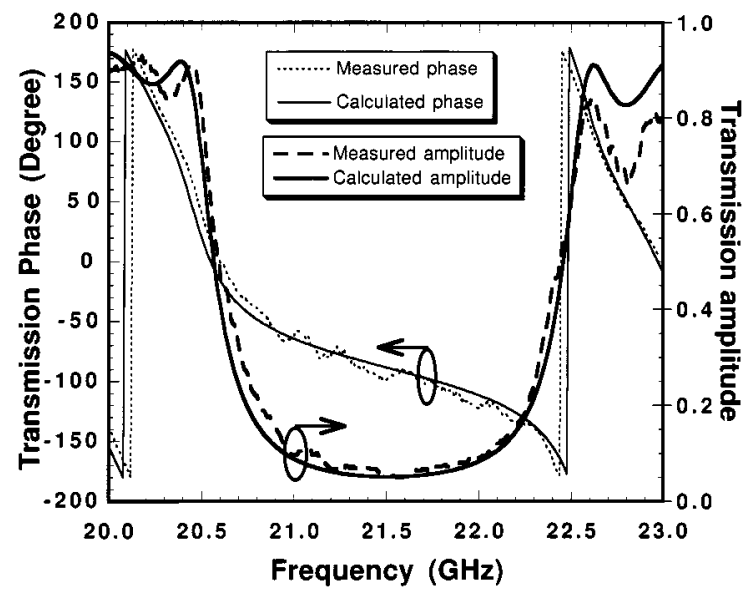

Fig. 3. Measured (thin dotted line) and calculated (thin solid line) transmission phase and measured (thick dashed line) and calculated (thick solid line) transmission amplitude for a DBR with $N=3$.

curves. The high value of the real part of the index for Eccostock allows one to use a minimal number of the slabs to obtain the desired response, further reducing the effects of propagation losses. On the other hand, compared to other materials such as polycarbonate, Plexiglass or Teflon, Eccostock demonstrates more inhomogeneity. This inhomogeneity can account for the small difference between the theoretical and experimental results. In passing, from Fig. 3, it should be clear that the assumption of constant phase for the transmission function is not a good one.

Finally, upon closer examination of Fig. 3, some small ripples in the measured transmission coefficient for the DBR is detected. Within the theory of operation for the NA, these small modulations are well understood and are attributed to the imperfect source/load mismatch [37]. Unfortunately, this effect cannot be removed by using the TRL calibration technique. For the purpose of the group delay and group velocity [see (9) and (11)], the phase of the transmission coefficient and not its magnitude is of most interest. Fig. 4 shows the calculated and measured unwrapped phase for DBR with three, two, and one dielectric slabs. As one may suspect, with increasing $N$, the finite DBR must approximate the infinite DBR more closely, and, in the limit of $N \rightarrow \infty$, the phase of the transmitted wave must approach a constant value. This trend is exhibited in Fig. 4.

To obtain the group delay, the curves depicted in Fig. 4 must be differentiated. The application of the differential operation to noisy data amplifies the noise and may lead to spurious effects. To reduce the noise, one may smooth the data prior to differentiation. However, smoothing the data prior to differentiation 


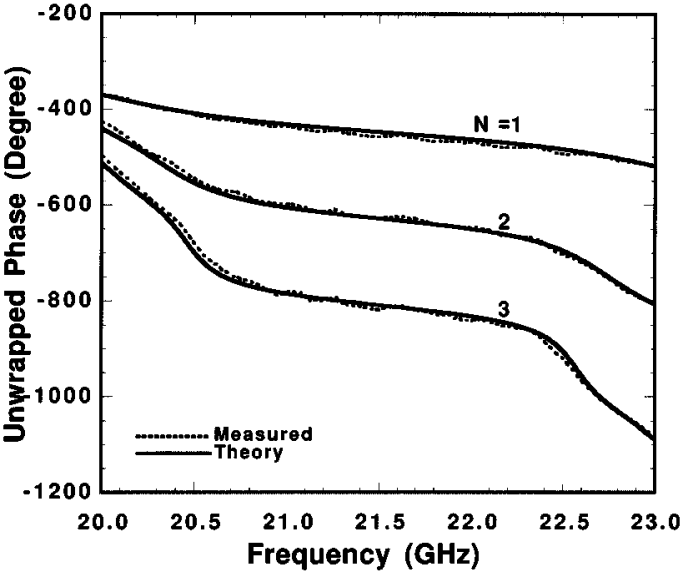

Fig. 4. Calculated (solid line) and measured (dotted line) unwrapped transmission phase.

is an arbitrary process that gives results which vary critically with the smoothing parameters. The approach used here is to obtain the best nonlinear least square fit to the experimental phase data. Since there is good agreement between theory and measurement (Fig. 4), and since the theoretical model [see (6)] for the transmission phase of the DBR is well established, the best least square fit to the data can be obtained by varying the dielectric thickness $\left(d_{j}\right)$, spacer thickness $\left(d_{i}\right)$, and the real part of the Eccostock index of refraction $\left(n_{j}^{\prime}\right)$. A Fortran program based on the IMSL subroutine DBCLSF, which uses a modified Levenberg-Marquardt algorithm and a finite-difference Jacobian, is used to obtain the best least square fit. Fig. 5 shows the result of the least square fit to the phase data of Fig. 4 together with applying (9) and (14) to determine the normalized group velocity of a DBR with one, two, and three dielectric slabs.

Along with the velocities derived from the fit (dotted curves), the theoretical group velocities calculated from measured values of the thicknesses and indices ${ }^{4}$ are also shown (solid curves). The fitting and measured values are give in Table I.

A closer examination of Fig. 5 reveals that, while one dielectric slab $(N=1)$ is insufficient to produce superluminal group velocities, two slabs are sufficient to set up the interferences resulting in a group velocity exceeding $c$. In the case of $N=3$, a maximum superluminal group velocity 2.1 times $c$ is observed. It is worth reemphasizing that in measurements with a VNA (frequency domain) such as the one described above, one can only measure the transmission coefficient (phase and magnitude) of quasi-CW sweeps. In another words, at no point in time has the VNA generated a microwave wave packet. In the next section, the sensitivity of the curves depicted in Fig. 5 to the experimental parameters will be discussed.

\section{Sensitivity ANALYsis}

Since the peak group velocity or normalized reciprocal group delay depicted in Fig. 5 occurs in the region of maximum attenuation of the transmitted signal, it is important to analyze

\footnotetext{
${ }^{4}$ The measured values of these variables were obtained by averaging several measurements. For example, the average value of the spacer thickness after 16 measurements was $1.76 \mathrm{~cm}$. The measured values for the indices and thicknesses were: $n_{i}=1.0, n_{j}=n \prime_{j}-i n \prime_{j}=3.40-i 0.002, d_{i}=1.76 \mathrm{~cm}, d_{j}=1.33$ $\mathrm{cm}$.
}

TABLE I

MEASURED AND FITTEd PARAMETRES FOR THE DBR DESCRIBED IN THE TEXT

\begin{tabular}{l|c|c|c|c}
\hline & Fitting, $N=3$ & Fitting, $N=2$ & Fitting, $N=1$ & Measured \\
\hline$d_{i}$ & $1.794 \mathrm{~cm}$ & $1.825 \mathrm{~cm}$ & - & $1.76 \mathrm{~cm}$ \\
\hline$d_{j}$ & $1.399 \mathrm{~cm}$ & $1.366 \mathrm{~cm}$ & $1.396 \mathrm{~cm}$ & $1.33 \mathrm{~cm}$ \\
\hline$n_{j}^{\prime}$ & 3.216 & 3.288 & 3.245 & 3.40 \\
\hline$n_{j}^{\prime \prime}$ & 0.002 & 0.002 & 0.002 & 0.002 \\
\hline
\end{tabular}

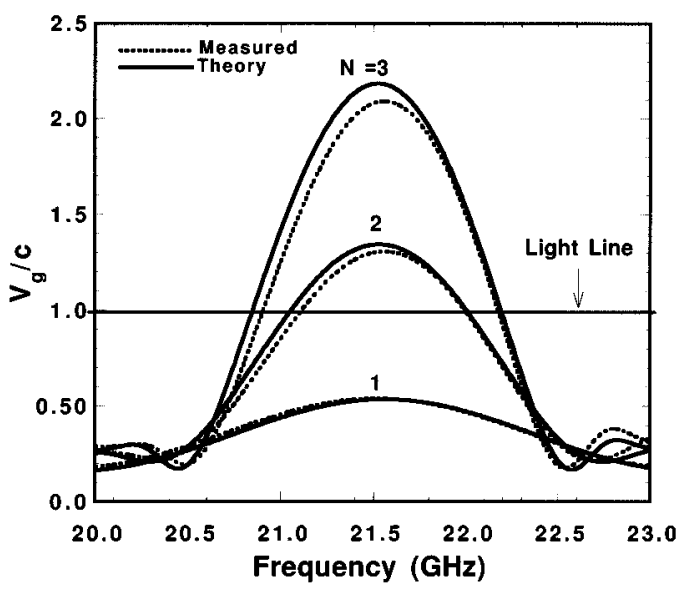

Fig. 5. Calculated (solid line) and fitted (dotted line) normalized group velocity (normalized reciprocal group delay) for a DBR with $N=3,2$, and 1 .

the experimental uncertainties involved. In general, the measured phase is a function of five variables: frequency $(v)$, spacer index $\left(n_{i}\right)$, spacer thickness $\left(d_{i}\right)$, dielectric-slab index $\left(n_{j}\right)$, and thickness $\left(d_{j}\right)$.

The HP-8722D VNA has a 1-Hz frequency resolution and an accuracy of $\pm 10 \mathrm{ppm}$ [35]. In the above experiment, the frequency interval between two adjacent points was set to $10 \mathrm{MHz}$, and the "step sweep" mode of the analyzer was used in order to obtain greater frequency accuracy. In addition, since the spacer is air, the spacer index $\left(n_{i}\right)$, is taken as unity. Therefore, the uncertainties due to the frequency and spacer index are negligible; however, the remaining three variables deserve closer attention.

In general, the variation of any function in terms of a given variable can be approximated by a difference equation. For example, variation of the normalized group velocity with respect to spacer thickness is given by

$$
\begin{aligned}
\Delta\left(V_{g} / c\right) & \approx \Delta d_{i} \frac{\partial\left(V_{g} / c\right)}{\partial d_{i}} \\
& =\Delta d_{i} \frac{2 \pi}{c(\partial \varphi / \partial v)^{2}}\left[(1-N) \frac{\partial \varphi}{\partial v}+L_{p c} \frac{\partial}{\partial d_{i}} \frac{\partial \varphi}{\partial v}\right]
\end{aligned}
$$

where $\partial \varphi / \partial v$ is proportional to the group delay. The expressions for the variation of the normalized group velocity (normalized reciprocal group delay) with respect to the other variables $\left(d_{j}, n_{j}\right)$ are similar to (17). In calculating the derivatives, it is assumed that the phase is a function of frequency and one other independent variable (e.g., $d_{i}$ ), while the other two variables (e.g., $n_{j}, d_{j}$ ) are assumed to be constant. A Fortran program using the IMSL routine DBS2DR was written to compute 


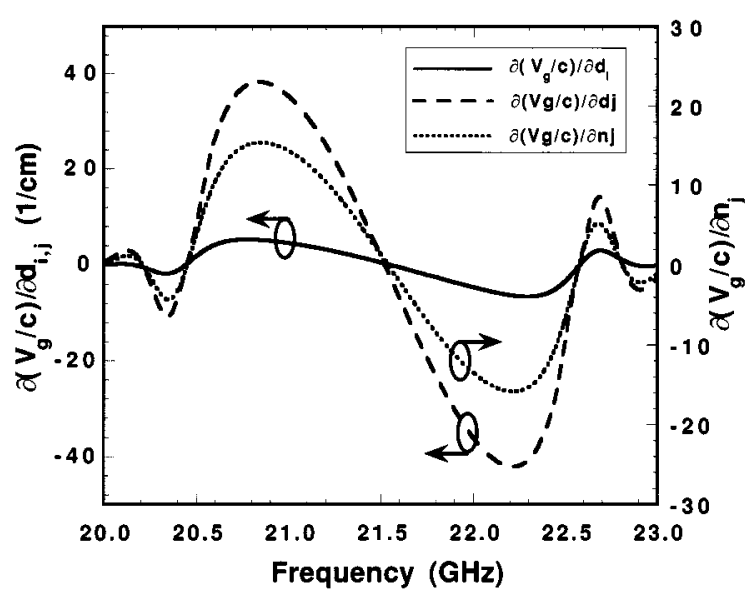

Fig. 6. Sensitivity of the normalized group velocity to the experimental parameters such as spacer thickness (solid line), dielectric-slab thickness (dashed line), and dielectric-slab index of refraction (dotted line).

the two-dimensional tensor-product spline interpolant and its derivative. The results for measured values of the variables, for the case of $N=3$, are depicted in Fig. 6.

From Fig. 6, it is evident that the experimental measurement is insensitive to uncertainty in parameters at "mid-gap" frequency or, equivalently, at the peak group velocity. As Fig. 6 indicates, the experimental group velocity is most sensitive to the dielectric-slab thickness with a maximum absolute value of 42.2 over the range of $20-23 \mathrm{GHz}$. On the other hand, using the phase-measurement technique described by (16) or by using other methods, accuracies up to $0.004 \mathrm{~cm}$ in measuring thicknesses are easily attainable. This means that the maximum absolute error in the curves depicted in Fig. 5 due to the dielectric-slab thickness is about 0.17. The maximum error over the range for which superluminal behavior is exhibited (approximately 21 to $22 \mathrm{GHz}$ ) is 0.14 . The experimental errors due to spacer thickness over the entire frequency range of $20-23 \mathrm{GHz}$ is even smaller, since the maximum absolute value of $\partial\left(v_{g} / c\right) / \partial d_{i}$ is 6.57 as compared to 42.2 .

Finally, the analysis of the experimental errors associated with the Eccostock index of refraction measurement is based on measuring the dielectric constant of $1.33 \mathrm{~cm}$ of air after the TRL calibration has been performed. These measurements indicated a maximum uncertainty in the Eccostock index of approximately 0.01 . Given that the maximum absolute value for $\partial\left(v_{g} / c\right) / \partial n_{j}$ over the frequency range $20-21 \mathrm{GHz}$ is 14.2 , experimental errors in the normalized group velocity of the order of 0.14 can be expected. Again, this error decreases to zero at the mid-gap. From the above analysis, it is clear that experimental errors are incapable of placing the normalized group velocity depicted in Fig. 5 below the light line.

A few remarks regarding the zeros of the derivatives shown in Fig. 6 are in order. These represent frequencies at which the sensitivity of the measured group velocity with respect to the experimental parameters is identically zero. From a physical point of view, the insensitivity at the "mid-gap" occurs because the DBR exhibits minimal dispersion at frequencies around $21.7 \mathrm{GHz}$. This minimizes the term in the brackets of (17). However, the zero crossings at the band edges occur because the group delay $(-\partial \varphi / 2 \pi \partial v)$ is very large and the denominator of the term outside the brackets in (17) dominates. From a technological point of view, the ability to design DBR's or delay lines for which the desired response is insensitive to variations in material parameters is of great importance. In fact, the inverse of the problem presented in this paper can be addressed by solving expressions such as (17) for the thickness and index of the dielectric slab and spacer, in order to design mirrors or delay lines that are less sensitive to the components' variations.

\section{CONCLUSIONS}

This paper describes the detection of superluminal group velocities for DBR in a free-space configuration. Diagonalization of the transfer matrix is used to obtain analytical closed-form expressions for both the transmission and reflection coefficients. Measuring a DBR in free space enables the removal of extraneous dispersion and dissipation and allows the detection of the superluminal group velocities. An experimental protocol based on the TRL calibration procedure is described resulting in good agreement between the experimentally measured and theoretically calculated phases. Accurate measurement of the transmission phase allows calculation of the group delay and generalized group velocity. A quantitative analysis of the possible errors indicates that the measured superluminal group velocities are, in fact, correct and in accordance with the theoretical models.

The ability to actually measure superluminal group velocities questions the traditional notion that, in regions near resonances, group velocity has "... no longer any appreciable physical significance" [30] or ".. ceases to have a clear physical meaning" [38]. Even up to last year, a generally respected text book such as "Classical Electrodynamics" by J. D. Jackson had considered the superluminal group velocities in the regions of anomalous dispersion as "...just not a useful concept" [39]. However, in the latest edition of this book (1998) while citing works by Chu and Wong [40] and Chiao [5], Jackson indicates that superluminal or negative group velocities "still have meaning" [41].

However, it must be emphasized that this superluminal behavior is in no way in contradiction with special relativity or causality. In fact, it can be shown that superluminal group velocities are the consequence of linear time-invariant systems which obey Kramers-Kronig relations and hence are inherently causal [5], [42]-[45], [26]. In the case of propagation through a DBR, the energy velocity remains subluminal [46], although this is not a universal requirement for the energy velocity [5], [45]. More importantly, under no circumstances does the front (the Sommerfeld forerunner) velocity exceed the speed of light in vacuum.

\section{REFERENCES}

[1] E. H. Hauge and J. A. Stovneng, "Tunneling times: A critical-review," Rev. Mod. Phys., vol. 61, pp. 917-936, 1989.

[2] L. A. MacDoll, "Note on the transmission and reflection of wave packets by potential barriers," Phys. Rev., vol. 40, pp. 621-626, 1932.

[3] E. P. Wigner, "Lower limit for the energy derivative of the scattering phase shift," Phys. Rev., vol. 98, pp. 145-147, 1955.

[4] T. E. Hartman, "Tunneling of a wave packet," J. App. Phys., vol. 33, pp. 3427-3433, 1962.

[5] R. Y. Chiao and A. M. Steinberg, "Tunneling times and superluminality," Prog. Opt., vol. 37, pp. 345-405, 1997. 
[6] T. Martin and R. Landauer, "Time-delay of evanescent electromagneticwaves and the analogy to particle tunneling," Phys. Rev. A, vol. 45, pp. 2611-2617, 1992.

[7] R. Y. Chiao, P. G. Kwiat, and A. M. Steinberg, "Analogies between electron and photon tunneling: A proposed experiment to measure photon tunneling times," Physica B, vol. 175, pp. 257-262, 1991.

[8] T. K. Gaylord, G. N. Henderson, and E. N. Glytsis, "Application of electromagnetics formalism to quantum-mechanical electron-wave propagation in semiconductors," J. Opt. Soc. Amer. B, vol. 10, pp. 333-339, 1993.

[9] D. I. Babic and S. W. Corzine, "Analytic expressions for the reflection delay; penetration depth; and absorptance of quarter-wave dielectric mirrors," IEEE J. Ouantum Electron., vol. 28, pp. 514-524, 1992.

[10] J. A. Lott, R. P. Schneider, K. J. Malloy, S. P. Kilcoyne, and K. D. Choquette, "Partial top dielectric stack distributed bragg reflectors for red vertical-cavity surface-emitting laser arrays," IEEE Photon. Technol. Lett., vol. 6, pp. 1397-1399, 1994.

[11] R. H. Yan, R. J. Simes, and L. A. Coldren, "Analysis and design of surface-normal fabry-perot electrooptic modulators," IEEE J. Quantum Electron., vol. 25, pp. 2272-2280, 1989.

[12] F. Koyama, Y. Suematsu, S. Arai, and T. E. Tawee, "1.5-1.6-Mu-M Galnasp/Inp dynamic-single-mode (Dsm) lasers with distributed bragg reflector,” IEEE J. Quantum Electron., vol. QE-19, pp. 1042-1051, 1983.

[13] A. Yariv, "Coupled-mode theory for guided-wave optics," IEEE J. Quantum Electron., vol. QE-9, pp. 919-933, 1973.

[14] N. Matuschek, F. X. Kartner, and U. Keller, "Analytical design of double-chirped mirrors with custom-tailored dispersion characteristics," IEEE J. Quantum Electron., vol. 35, pp. 129-137, 1999.

[15] A. M. Steinberg, P. G. Kwiat, and R. Y. Chiao, "Measurement of the single-photon tunneling time," Phys. Rev. Lett., vol. 71, pp. 708-711, 1993.

[16] C. Spielmann, R. Szipocs, A. Stingl, and F. Krausz, "Tunneling of optical pulses through photonic band-gaps," Phys. Rev. Lett., vol. 73, pp. 2308-2311, 1994.

[17] A. Ranfagni, D. Mugnai, P. Fabeni, and F. Krausz, "Delay-time measurements in narrowed wave-guides as a test of tunneling," Appl. Phys. Lett., vol. 58, pp. 774-776, 1991.

[18] A. Ranfagni, P. Fabeni, G. P. Pazzi, and D. Mugnai, "Anomalous pulse delay in microwave propagation: A plausible connection to the tunneling time," Phys. Rev. E, vol. 48, pp. 1453-1460, 1993.

[19] D. Mugnai, A. Ranfagni, and L. Ronchi, "The question of tunneling time duration: A new experimental test at microwave scale," Phys. Lett. A, vol. 247, pp. 281-286, 1998.

[20] A. Enders and G. Nimtz, "Photonic-tunneling experiments," Phys. Rev. $B$, vol. 47, pp. 9605-9609, 1993.

[21] — " "On superluminal barrier traversal," J. Phys. I France, vol. 2, pp. 1693-16988, 1992.

[22] — "Evanescent-mode propagation and quantum tunneling," Phys. Rev. E, vol. 48, pp. 632-634, 1993.

[23] — , "Zero-time tunneling of evanescent mode packets," J. Phys. I France, vol. 3, pp. 1089-1092, 1993.

[24] G. Nimtz, A. Enders, and H. Spieker, "Photonic tunneling times," J. Phys. I France, vol. 4, pp. 565-570, 1994.

[25] G. Nimtz and W. Heitmann, "Superluminal photohnic tunneling and quantum-electronics," Prog. Quantum Electron., vol. 21, pp. 81-108, 1997.

[26] M. Mojahedi, "Superluminal group velocities and structural dispersion," Dissertation in EECE, University of New Mexico, 1999.

[27] D. J. Kane, "Recent progress toward real-time measurement of ultrashort laser pulses," IEEE J. Quantum Electron., vol. 35, pp. 421-431, 1999.

[28] R. Trebino and D. J. Kane, "The dilemma of ultrashort-laser-pulse intensity and phase measurement and applications," IEEE J. Quantum Electron., vol. 35, pp. 418-420, 1999.
[29] M. V. Klein and T. E. Furtak, Optics, 2nd ed. New York: Wiley, 1986.

[30] M. Born and E. Wolf, Principles of Optics; Electromagnetic Theory of Propagation, Interference and Diffraction of Light, 4th ed. New York, NY: Pergamon Press, 1970.

[31] J. P. Dowling, "Parity; time-reversal and group delay for inhomogeneous dielectric slabs: Application to pulse propagation in finite; one-dimensional; photonic bandgap structures," Proc. Inst. Elect. Eng., pt. J, vol. 145, pp. 420-435, 1998.

[32] G. Strang, Linear Algebra and Its Applications, 3rd ed. Orlando, FL: Harcourt Brace Jovanovich, 1988.

[33] A. Papoulis, The Fourier Integral and Its Applications. New York, NY: McGraw-Hill, 1962.

[34] L. Brillouin, Wave Propagation and Group Velocity. New York, NY: Academic, 1960.

[35] Hewlett Packard, HP 8719D/8720D/8722D network analyzer, in User's Guide 08720-90 288, 1997.

[36] Hewlett Packard, Applying the HP 8510 TRL calibration for noncoaxial measurements, in Product Note 8510-8A, 1992.

[37] Hewlett Packard, Measuring dielectric constant with the HP 8510 network analyzer, in Product Note 8510-3, 1985.

[38] L. Brillouin, Wave Propagation in Periodic Structures; Electric Filters and Crystal Lattices, 1st ed. New York, NY, London: McGraw-Hill, 1946.

[39] J. D. Jackson, Classical Electrodynamics, 2nd ed. New York, NY: Wiley, 1975

[40] S. Chu and S. Wong, "Linear pulse-propagation in an absorbing medium," Phys. Rev. Lett., vol. 48, pp. 738-741, 1982.

[41] J. D. Jackson, Classical Electrodynamics, 3rd ed. New York, NY: Wiley, 1998

[42] M. D. Crisp, "Concept of group velocity in resonant propagation," Phys. Rev. A, vol. 4, pp. 2104-2108, 1971.

[43] E. L. Bolda, R. Y. Chiao, and J. C. Garrison, "Two theoremd for the group velocity in dispersive media," Phys. Rev. A, vol. 48, pp. 3890-3894, 1993.

[44] G. Diener, "Superluminal group velocities and information-transfer," Phys. Lett. A, vol. 223, pp. 327-331, 1996.

[45] R. Y. Chiao, J. Boyce, and M. W. Mitchell, "Superluminality and parelectricity: The ammonia maser revisited," Appl. Phys. B, vol. 60, pp. 259-265, 1995.

[46] M. Scalora, J. P. Dowling, A. S. Manka, C. M. Bowden, and J. W. Haus, "Pulse-propagation near highly reflective surfaces: Applications to photonic band-gap structures and the question of superluminal tunneling times," Phys. Rev. A, vol. 52, pp. 726-734, 1995.

Mohammad Mojahedi, photograph and biography not available at the time of publication.

Edl Schamiloglu, photograph and biography not available at the time of publication.

Kamil Agi, photograph and biography not available at the time of publication.

Kevin J. Malloy, photograph and biography not available at the time of publication. 